\title{
Let-7a inhibits migration, invasion and epithelial- mesenchymal transition by targeting HMGA2 in nasopharyngeal carcinoma
}

Aibing $\mathrm{Wu}^{\dagger}$, Kunpeng $\mathrm{Wu}^{*}{ }^{*}$, Jinmei Li ${ }^{\dagger}$, Yanli Mo, Yanming Lin, Yuzhou Wang, Xiang Shen, Shujun Li, Lixia Li and Zhixiong Yang

\begin{abstract}
Background: Let-7a has been shown to play important roles in nasopharyngeal carcinoma (NPC) cell proliferation and apoptosis, but little is known about the function and mechanism of let-7a in nasopharyngeal carcinoma metastasis. We aimed to investigate the function and mechanism of let-7a in nasopharyngeal carcinoma metastasis and clarified the regulation of high mobility group A2 (HMGA2) by let-7a.

Methods: The expression levels of let-7a and HMGA2 were examined in NPC clinical specimens using quantitative reverse transcription-PCR (RT-qPCR). HMGA2 was confirmed as a target of let-7a through luciferase reporter assays, RT-qPCR, and Western blotting. Furthermore, the roles of let-7a and HMGA2 in regulating NPC cells biological properties including proliferation, migration, invasion and epithelial-mesenchymal transition (EMT) process were analyzed with let-7a mimics and si-HMGA2 transfected cells.
\end{abstract}

Results: Our study demonstrated that let-7a was downregulated and inversely associated with the clinical stage, $T$ classification and N classification, and HMGA2 was upregulated and directly associated with the clinical stage and $\mathrm{N}$ classification in patients with NPC. Moreover, there was an inverse correlation between let-7a expression and HMGA2 expression in NPC patient. In addition, HMGA2 was negatively regulated at the posttranscriptional level by let-7a via a binding site of HMGA2-3'UTR. In addition, synthetic let-7a mimics suppressed NPC cells migration, invasion and EMT process and knockdown of HMGA2 was consistent with the effects of let-7a in NPC cells.

Conclusion: Let-7a directly downregulates HMGA2 protein expression, which suppress NPC cell migration, invasion and EMT process. Let-7a could serve as a potential diagnostic marker and therapeutic target for NPC.

Keyword: MicroRNA, Let-7a, HMGA2, Nasopharyngeal carcinoma, Metastasis

\section{Background}

Nasopharyngeal carcinoma (NPC) is a malignant disease with a distinct geographical and ethnic distribution. The global statistics of cancer showed its distinctly unbalanced endemic distribution, with the highest incidence in Southern China [1]. Unfortunately, the majority of NPC patients tend to present a more advanced stage of disease when first diagnosed because of its vague symptoms and deep location. Although NPC patients are

\footnotetext{
*Correspondence: 67104234@163.com; yangzhixiong068@126.com ${ }^{\dagger}$ Equal contributors

Oncology Center, Affiliated Hospital of Guangdong Medical College, No.57 Peoples Avenue South, Zhanjiang, Guangdong 524002, People's Republic of
} China

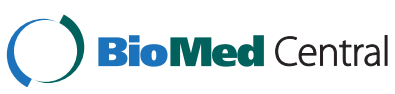

(c) 2015 Wu et al.; licensee BioMed Central. This is an Open Access article distributed under the terms of the Creative Commons Attribution License (http://creativecommons.org/licenses/by/4.0), which permits unrestricted use, distribution, and reproduction in any medium, provided the original work is properly credited. The Creative Commons Public Domain Dedication waiver (http://creativecommons.org/publicdomain/zero/1.0/) applies to the data made available in this article, unless otherwise stated. sensitive to radiotherapy, treatment failure remains high due to the development of local recurrence and distant metastasis [2]. Because the mechanisms of NPC metastasis that are not completely understood to date, further investigation of this mechanism is imminently needed.

MicroRNAs (miRNAs) are endogenous non-coding small RNAs about 19-25 nucleotide long, which contribute to the regulation of their target genes mRNA by usually base-pairing to the 3 '-untranslated region $\left(3^{\prime}\right.$ UTR), and results in either mRNA degradation or translation inhibition [3-5]. It has been reported that miRNAs can control a variety of biological processes including cellular differentiation, proliferation, cell mobility and cell death [6,7]. Moreover, recent evidence indicated that 
miRNAs can function either as tumor suppressors or oncogenes in tumor progression $[8,9]$. Recent studies reported that several miRNAs are related to NPC development and progression by regulating cell growth, metastasis, and apoptosis [10-13], indicating that miRNAs play significant roles in NPC tumorigenesis. However, no study has elucidated the functions and mechanisms of let-7 miRNA family in NPC metastasis.

Let-7 was first identified in Caenorhabditis elegans and is highly conserved in C.elegans, Drosophila, Zebrafish, and Humans [3]. Let-7 scarcely expresses in embryonic stages, but obviously enhances in mature and differentiated tissues [14]. Moreover, let-7 has been shown as being decreased during cancer progression in various human cancers [3]. Recent studies have indicated that let-7 regulated expression of certain oncogenes and contributed to carcinogenesis, in lung [15], colon [16], head and neck [17], and pancreatic cancer [18]. In addition, several studies further revealed that let-7 plays important roles in the regulation of metastasis and epithelialmesenchymal transition (EMT) in breast cancer [19], oral cancer [20] and esophageal cancer [21]. Interestingly, downregulation of let-7 initiated and maintained oncostatin M-induced EMT phenotypes in breast cancer, and that high mobility group A2 (HMGA2) acted as a switching actor in this progress [19].

HMGA2 is a small nonhistone chromosomal protein that has no intrinsic transcriptional activity, but can modulate transcription by altering chromatin architecture [22]. HMGA2 proteins are involved in many diverse biological processes such as differentiation, embryogenesis, and neoplastic transformation [23]. In contrast with let-7, HMGA2 was overexpressed in embryonic and carcinoma tissue and was associated with highly malignant phenotype and was a poor prognostic factor [24]. Three miRNAs target databases (TargetScan, miRanda and PicTar) predicted HMGA2 to be a potential target of let-7. Although the direct regulatory relationship between let-7a and HMGA2 has been confirmed in lung cancer [25], breast cancer [19], and esophageal cancer [21], little is known about let-7 and HMGA2 in NPC.

In this study, we aimed to investigate the role of let-7a and HMGA2 in NPC. We found that downregulation of let-7a and upregulation of HMGA2 in NPC tissues compared with normal tissues, and associated with clinical stage and N classification in NPC. Moreover, there was an inverse correlation between let-7a expression and HMGA2 expression in NPC patient. In addition, synthetic let-7a mimics inhibited the migration, invasion, and EMT of NPC cells in vitro. Furthermore, we validated HMGA2 was a target of let-7a by 3 '-UTR luciferase assays and western blot analysis. Meanwhile, knockdown of HMGA2 was consistent with the effects of let-7a in NPC cells, but no influence on the expression of let-7a. Our study demonstrated that let-7a targets HMGA2 to regulate migration and invasion through epithelial-mesenchymal transition in NPC.

\section{Materials and methods Clinical specimens}

A total of 48 freshly-frozen NPC samples and 20 normal nasopharyngeal epithelium samples were collected from the Affiliated Hospital of Guangdong Medical School between March 2013 and March 2014. No patients had received any form of tumour-specific therapy before diagnosis. Before the use of these clinical samples, prior consents from the patients and approval from the Institutional Ethics Committee of the Affiliated Hospital of Guangdong Medical School were obtained (HM-81201672). The histopathological diagnosis of all samples was respectively diagnosed by two pathologists. The clinical staging was based on the 7th edition of the AJCC Cancer Staging Manual. The main demographic and clinicopathological characteristics were presented in Table 1.

\section{Cell culture}

All other cell lines were from the Cell Bank of Type Culture Collection of the Chinese Academy of Sciences (Shanghai, China). Five NPC cell lines HONE1, CNE1, CNE2, 5-8F and 6-10B were maintained in RPMI 1640 medium supplemented with $10 \%$ fetal bovine serum (FBS; Biological Industries, Israel). All the cell lines were incubated in a humidified chamber with $5 \% \mathrm{CO}_{2}$ at $37^{\circ} \mathrm{C}$, and used for this study within 6 months of resuscitation.

\section{Real-time quantitative reverse transcription-polymerase chain reaction ( $\mathrm{RT}-\mathrm{qPCR}$ )}

To quantitate mRNA expression, total RNA was extracted from clinical samples and NPC cell lines with RNAiso Plus (Takara, Japan). The isolated total RNA was reverse transcribed using the One Step PrimeScript miRNA cDNA Synthesis Kit (Takara, Japan) for Let-7a and the PrimeScript RT Master Mix (Perfect Real Time) (Takara, Japan) for HMGA2, according to manufacturer instructions. Relative expression was calculated via the comparative cycle threshold $(\mathrm{Ct})$ method and was normalized to the expression of U6 small RNA or $\beta$-actin. The sequence-specific forward primers for mature Let-7a and U6 internal control were 5'-GGTGAGGTAGTAGGTTG TATAGTT-3' and 5'-CTCGCTTCGGCAGCACATATA3', respectively. The Uni-miR qPCR Primer was included in the kit. Forward and reverse primers sequences for HMGA2 mRNA were 5' - CTCAAAAGAAAGCAGAAGC CACTG - 3 ' and 5' - TGAGCAGGCTTCTTCTGAACAA $\mathrm{CT}-3$ respectively. Forward and reverse primers sequences for $\beta$-actin mRNA were $5^{\prime}$-GGCGGCAACACCATGTAC CCT-3' and 5' -AGGGGCCGGACTCGTCATACT-3 respectively. qPCR was performed using SYBR Premix Ex 


\begin{tabular}{|c|c|c|c|c|c|c|c|}
\hline \multirow[t]{2}{*}{ Characteristics } & \multirow[t]{2}{*}{$\mathrm{n}$} & \multicolumn{2}{|l|}{ Let-7a } & \multirow[t]{2}{*}{$p$} & \multicolumn{2}{|l|}{ HMGA2 } & \multirow[t]{2}{*}{$p$} \\
\hline & & High, $n(\%)$ & Low, n(\%) & & High, n(\%) & Low, n(\%) & \\
\hline \multicolumn{8}{|l|}{ Gender } \\
\hline Male & 28 & 14(50.0) & $14(50.0)$ & 1.000 & $12(42.9)$ & 16(57.1) & 0.063 \\
\hline Female & 20 & $10(50.0)$ & $10(50.0)$ & & 14(70.0) & $6(30.0)$ & \\
\hline \multicolumn{8}{|l|}{ Age(y) } \\
\hline$\geq 50$ & 24 & $11(45.8)$ & $13(54.2)$ & 0.564 & $10(41.7)$ & $14(58.3)$ & 0.082 \\
\hline$<50$ & 24 & $13(54.2)$ & $11(45.8)$ & & $16(66.7)$ & $8(33.3)$ & \\
\hline \multicolumn{8}{|l|}{ Clinical stage } \\
\hline$|-| \mid$ & 16 & $12(75.0)$ & $4(25.0)$ & 0.014 & $5(31.2)$ & $11(68.8)$ & 0.024 \\
\hline III-IV & 32 & $12(37.5)$ & $20(62.5)$ & & $21(65.6)$ & 11(34.4) & \\
\hline \multicolumn{8}{|l|}{ T classification } \\
\hline $\mathrm{T} 1-\mathrm{T} 2$ & 21 & 15(71.4) & $6(28.6)$ & 0.009 & $9(42.9)$ & $12(57.1)$ & 0.165 \\
\hline T3-Т4 & 27 & $9(33.3)$ & $18(66.7)$ & & 17(63.0) & 10(37.0) & \\
\hline \multicolumn{8}{|l|}{ N classification } \\
\hline NO-N1 & 19 & 13(68.4) & $6(31.6)$ & 0.039 & $5(26.3)$ & $14(73.7)$ & 0.002 \\
\hline N2-N3 & 29 & $11(37.9)$ & $18(62.1)$ & & $21(72.4)$ & $8(27.6)$ & \\
\hline \multicolumn{8}{|c|}{ Distant metastasis } \\
\hline No & 44 & $24(54.5)$ & $20(45.5)$ & 0.117 & 23(52.3) & $21(47.7)$ & 0.727 \\
\hline Yes & 4 & $0(0)$ & $4(100)$ & & $3(75.0)$ & $1(25.0)$ & \\
\hline
\end{tabular}

Taq $^{\text {Ts }}$ II (Takara, Japan) on a LightCycler (Roche Diagnostics, USA). Relative quantification of miRNA expression was calculated by using the $2^{-\Delta \mathrm{Ct}}$ method. The raw data were presented as the relative quantity of target miRNA or mRNA, normalized with respect to U6 or $\beta$ actin, and relative to a calibrator sample. All qRT-PCR reactions were performed in triplicate.

\section{miRNAs, small interfering RNAs and transfection}

Let-7a mimic and the negative control were obtained from RiboBio (Guangzhou, China). Small interfering RNAs (siRNAs), HMGA2-siRNA 1, HMGA2-siRNA 2, HMGA2-siRNA 3 and the negative control siRNA were also obtained from RiboBio (Guangzhou, China). For convenience, Let-7a mimic negative control and negative control siRNA are termed Let-7a mimics-NC and NCsiRNA. miRNAs and siRNAs transfection was performed using riboFECT ${ }^{\mathrm{T} m} \mathrm{CP}$ (RiboBio, China) according to the manufacturer's instruction. The relative level of Let-7a and HMGA2 in transfected cells were examined by qRT-PCR. (Total RNAs and protein were prepared $48 \mathrm{~h}$ after transfection for RT-qPCR and western blotting analysis.)

\section{Luciferase reporter assays}

The HMAGA2 wild-type (Wt) and mutant (Mt) 3'UTR were created and cloned to the firefly luciferase-expressing vector pLUC (Additional file 1: Figure S2). For the luciferase assay, CNE-2 and 5-8F cells were seeded triplicate in 12-well plates the day before transfection, and cotransfected with the HMAGA2 Wt or Mt 3'UTR reporter vector, and Let-7a mimics or Let-7a mimics-NC using riboFECT ${ }^{\mathrm{im}} \mathrm{CP}$ (RiboBio, China). After 48 hours of transfection, the cells were harvested and lysed, and the luciferase activities were assayed using the Dual-Luciferase Reporter System (Promega, USA). Three independent experiments were performed.

\section{Cell proliferation assay}

Cell proliferation was analyzed using MTT assay. Briefly, $1 \times 10^{3}$ cells were seeded into a 96-well plate with quadruplicate repeat for each condition. For si-MMP14 and si-control, the cells were incubated for 1, 2, 3, and 4 days. Twenty microliters of MTT $(5 \mathrm{mg} / \mathrm{ml}$ ) (Sigma, USA) was added to each well and incubated for $4 \mathrm{~h}$. At the end of incubation, the supernatants were removed and $150 \mu \mathrm{l}$ of DMSO (MP, USA) was added to each well. The absorbance value (OD) of each well was measured at $490 \mathrm{~nm}$. Experiments were performed three times.

\section{Cell migration and invasion assays}

After $48 \mathrm{~h}$ transfection, cells were resuspended into serum-free medium. For migration assays, $5.0 \times 10^{4}$ cells were placed in the top chamber of each insert (Corning 

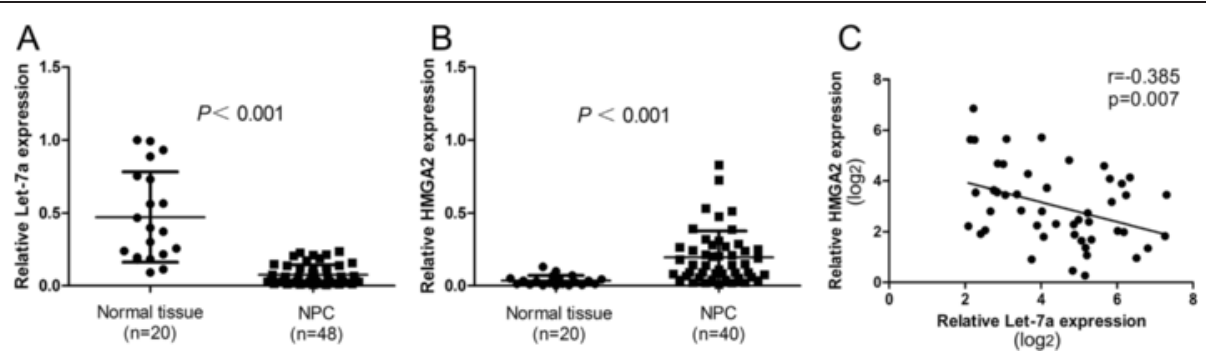

Figure 1 Expression of let-7a and HMGA2 in NPC clinical samples. (A-B) Let-7a was downregulated and HMGA2 was upregulated in NPC $(n=48)$ compared with normal nasopharyngeal epithelial tissues $(n=20)$. Data is presented as the mean $\pm S D$, and P values were calculated with the Student $t$-test. (C) Significant correlations between the expression of let-7a and HMGA2 in NPC $(n=48)$ were demonstrated using the Pearson's correlation coefficient analysis $(r=-0.385, P=0.007)$.

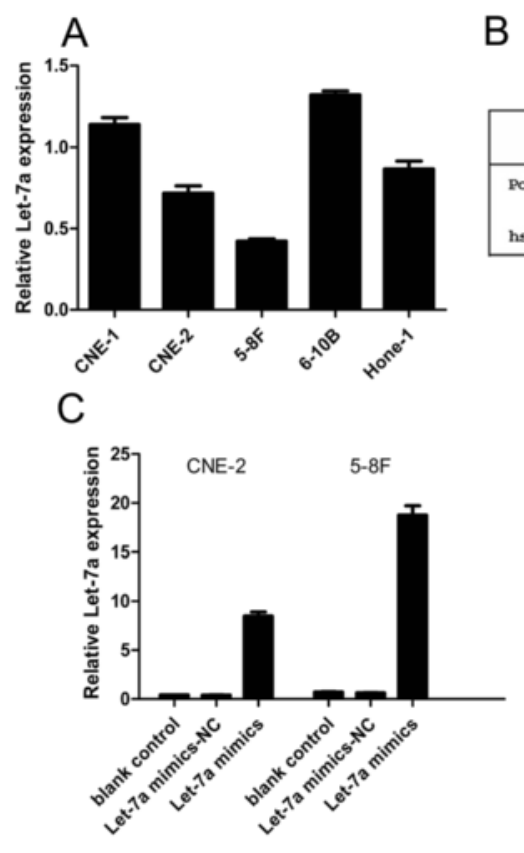

B

E
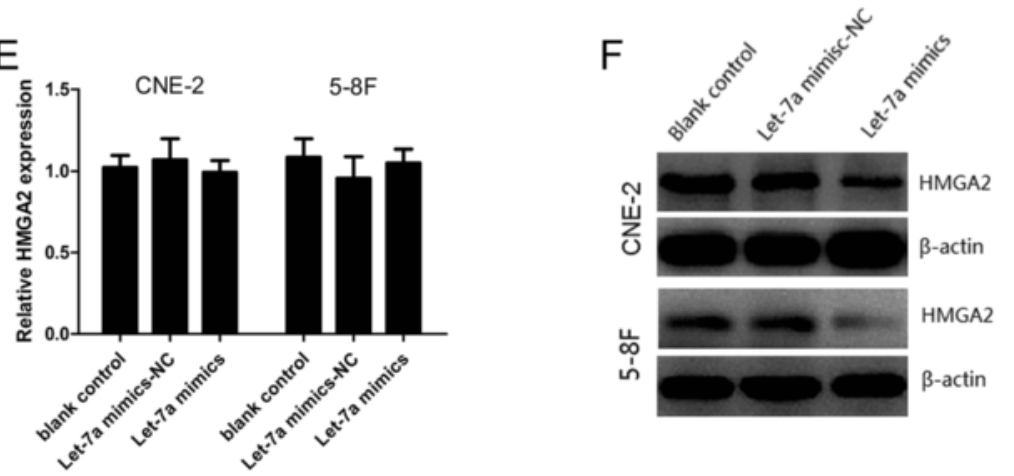

Figure 2 Let-7a inhibited the protein expression of HMGA2 by binding to its $\mathbf{3}^{\prime} \mathbf{U T R}$. (A) RT-qPCR of let-7a expression in NPC cell lines with different degrees of differentiation and metastasis ability. (B) CNE-2 and 5-8F cells transfected with the let-7a mimics showed significantly increased let-7a expression compared to controls. (C) Position of the let-7a target site in 3'UTR of HMGA2 mRNA predicted by TargetScan. (D) Relative luciferase activity of CNE-2 and 5-8F cells after co-transfection with wild type (WT) or mutant (MUT) HMGA2 3'UTR reporter genes and let-7a mimics or control. (E-F) Protein and mRNA expression levels of HMGA2 are tested after let-7a transfected in CNE-2 and 5-8F by western blotting and RT-qPCR. ${ }^{*}, P<0.001$ compared with control. 
Costar, USA) with $8.0 \mu \mathrm{m}$ pores; for invasion assays, $1.0 \times 10^{5}$ cells were seeded in a Matrigel-coated chamber (Corning Costar, USA). In the lower chamber, $600 \mu \mathrm{l}$ of RPMI 1640 with 10\% FBS was added as a chemoattractant. After the cells were incubated for $24 \mathrm{~h}$, the insert was washed with PBS, and cells on the upper surface of the membrane were removed with a cotton swab. Cells adhering to the lower surface were fixed with methanol, stained with Giemsa. The number of cells in the membrane was counted from 5 randomly selected visual fields with a microscope at $100 \times$ magnification. All assays were independently repeated at least three times.

\section{Western blotting}

Transfected CNE-2 and 5-8F cells were cultured for 72 hours and then harvested on ice using RIPA lysis buffer
(Cwbiotech, China). Total protein concentrations were measured using the BCA protein assay kit (Cwbiotech, China). Total protein was separated by SDS-PAGE using 8-12\% polyacrylamide gel and transferred to polyvinylidenefluoride membrane (PVDF; Millipore, USA). The membrane was immunoblotted overnight at $4{ }^{\circ} \mathrm{C}$ with primary antibodies: anti-HMGA2, anti-E-Cadherin, anti-ZO-1, anti-MMP-2, anti-MMP-9, anti-Vimentin, anti-Snail, Slug, anti- $\beta$-Catenin(1:1000 dilution; Cell Signaling Technology, USA), and anti- $\beta$-actin (1:2000 dilution; Cwbiotech, China). The secondary antibody, horseradish peroxidaseconjugated goat IgG (1:1000 dilution; Cell Signaling Technology, USA), was incubated with the membrane for $1 \mathrm{~h}$ after 3 washes with TBST. Signals were detected with ECL detection reagent (Cwbiotech, China). The images were obtained and quantified by Quantity One (Bio-Rad, USA). Each experiment was performed in triplicates.
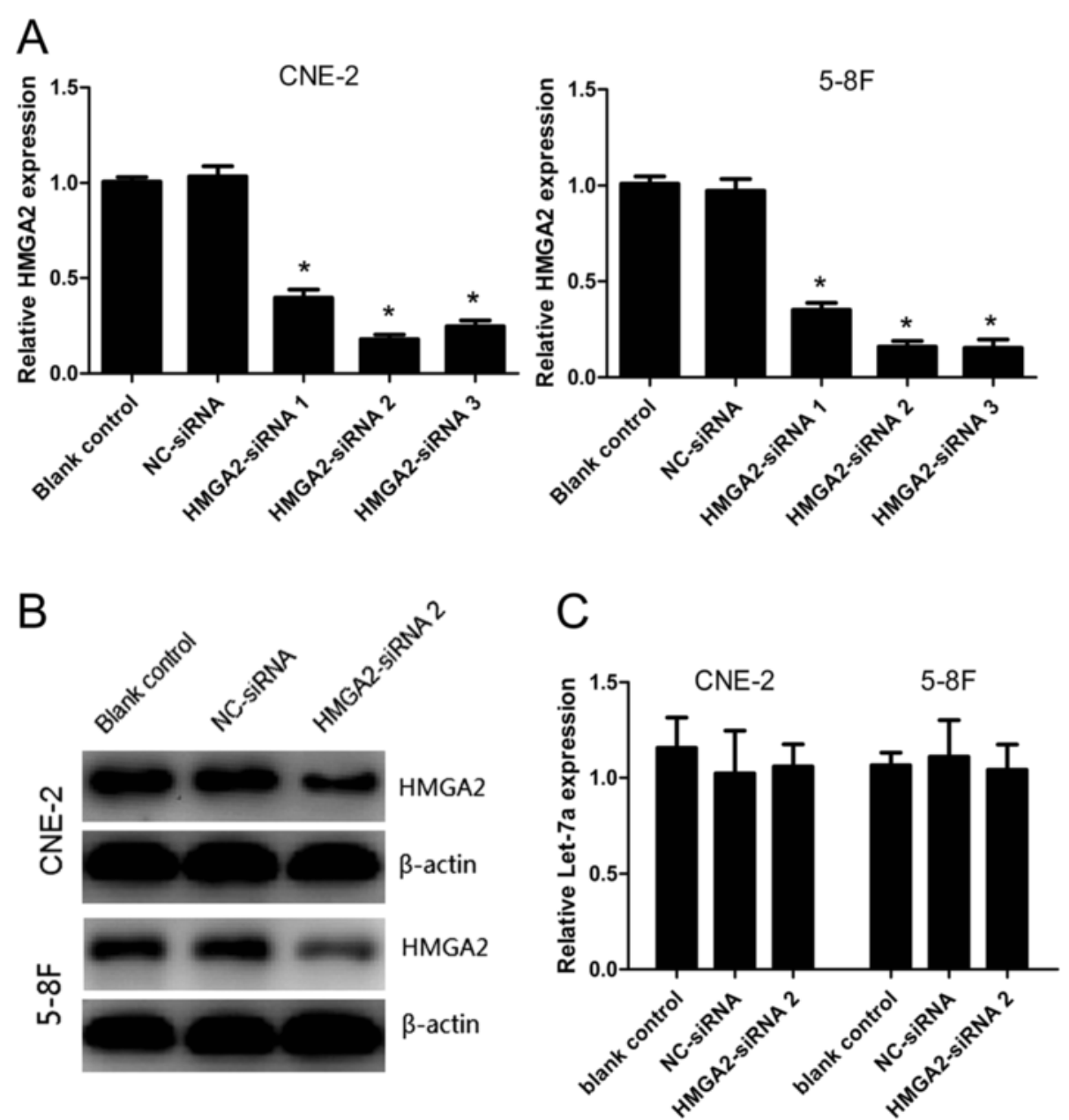

Figure 3 HMGA2 is regulated uniaxially by Let-7a but not feedback regulatory loop. (A) The efficiency of HMGA2-siRNA was distinguished by RT-qPCR in NPC cells and HMGA2 si-RNA 2 was most effective both CNE-2 and 5-8F cells. (B) Analysis of protein expression by western blot, HMGA2 protein is reduced by small interfering RNA (HMGA2-siRNA 2) in CNE-2 and 5-8F cells. (C) Knocking down the expression of HMGA2 had no alternation on let-7a level. *, $\mathrm{P}<0.001$ compared with control. 
A
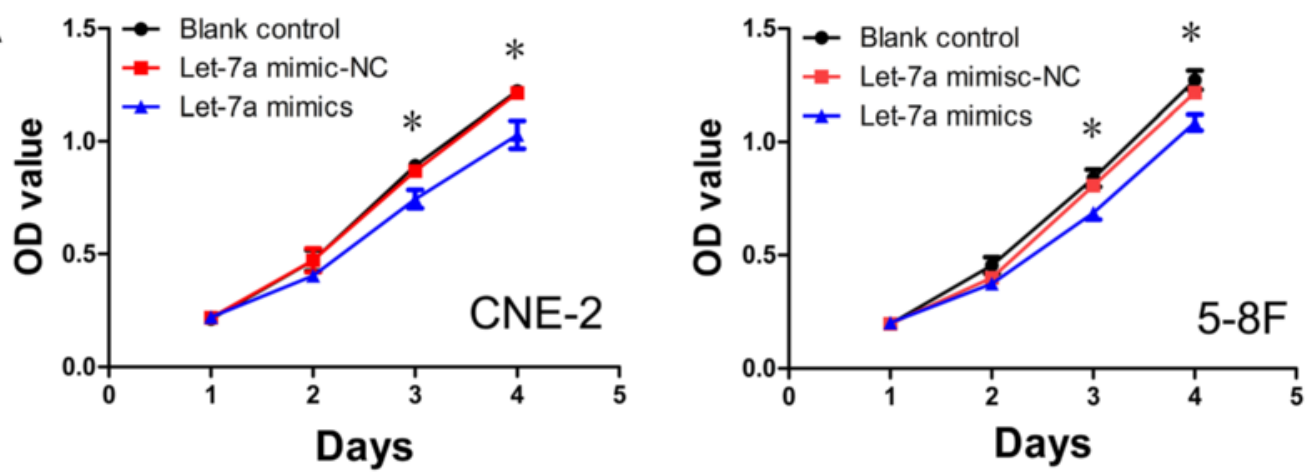

B

Blank control
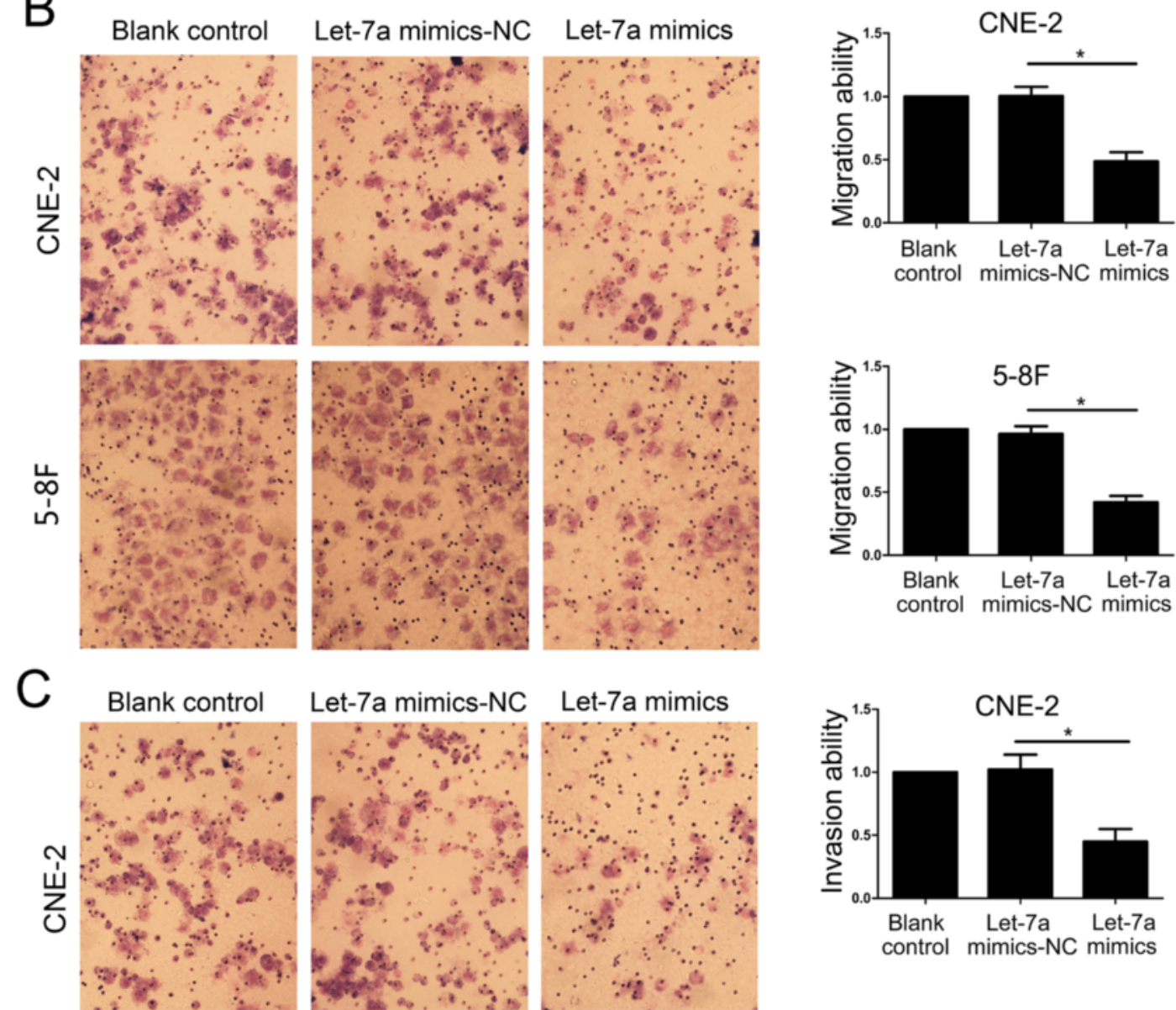

Let-7a mimics-NC
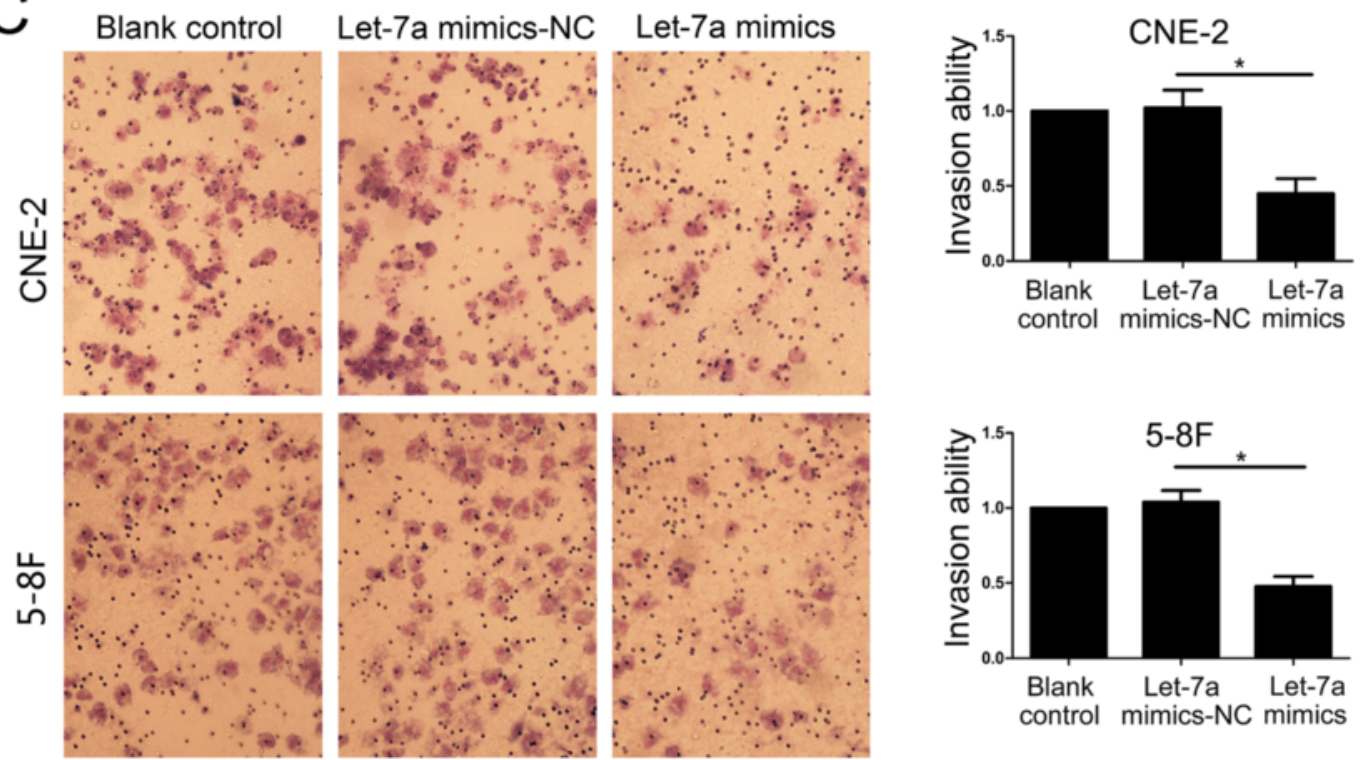

Figure 4 (See legend on next page.) 
(See figure on previous page.)

Figure 4 Overexpression of let-7a suppressed NPC cells proliferation, migration and invasion. (A) The growth curves determined by MTT assay showed that let-7a mimics inhibited NPC cells growth compared with negative control of let-7a. (B) Up-regulated let-7a expression dramatically decreased the ability of CNE-2 and 5-8F cells migration in vitro. (C) Elevated let-7a expression inhibited invasiveness of CNE-2 and $5-8$ F cells. The results were expressed as fold change relative to the corresponding blank control. Data is presented as the mean \pm SD. ${ }^{*}, P<0.001$ compared with control.

\section{Statistical analysis}

Data were presented as mean $\pm \mathrm{SD}$. The Student's $t$ test was used for comparisons of two independent groups. One-way ANOVA was used to determine cell growth in vitro. The Chi-square test was applied to the examination of relationship between let-7a and HMGA2 expression and clinicopathologic characteristics. All statistical analysis was performed with SPSS 17.0 software, and $P$ values of $<0.05$ were defined as statistically significant.

\section{Results}

Let-7a was downregulated and HMGA2 was upregulated in NPC clinical specimens

In this study, we firstly tested let-7a expression in 48 freshly-frozen NPC and 20 normal nasopharyngeal tissue samples. Compared with normal nasopharyngeal epithelial tissues, NPC tissues showed lower expression levels of let-7a and higher expression levels of HMGA2 mRNA. (Figure 1A-B, both $P<0.001$ ).

\section{Relationship between clinicopathological characteristics} and the expression of let-7a and HMGA2 in NPC patients In this study, patients with values less than the average expression level of let-7a and HMGA2 were assigned to a low expression group, whereas those with expression values above average were assigned to a high expression group. The cut-off levels were 4.41 for let-7a (normalized to U6), and 3.01 for HMGA2 (normalized to $\beta$ actin), which is the mean level of relative quantity. This classification was based on published studies [26,27]. The relationships between clinicopathological characteristics and let-7a and HMGA2 expression levels in individuals with NPC are summarized in Table 1. We observed that the expression level of let-7a was positively correlated with the status of clinical stage (I-II vs. III-IV, $P=0.014$ ), T classification (T1-T2 vs. T3-T4, $P=0.009$ ), and N classification (N0-N1 vs. N2-N3, $P=0.039$ ) in NPC patients. However, we did not find a significant association of let-7a expression levels with patient's gender (Male vs. Female, $P=1.000$ ), age ( $\geq 50$ vs. $<50, P=0.564$ ), and distant metastasis (Yes vs. No, $P=0.117$ ). In addition, there were significant correlations between HMGA2 expression and clinical staging (I-II vs. III-IV, $P=0.024$ ), and N classification (N0-N1 vs. N2-N3, $P=0.002$ ) in NPC patients. However, HMGA2 expression was not associated significantly with gender (Male vs. Female, $P=0.063$ ), age ( $\geq 50$ vs. $<50$,
$P=0.082)$, T classification (T1-T2 vs. T3-T4, $P=0.165)$, distant metastasis (Yes vs. No, $P=0.727$ ).

Inverse correlation between let-7a expression and HMGA2 expression in NPC patients

In $48 \mathrm{NPC}$ patients, the inverse correlation between the expression of let-7 and HMGA2 in NPC was confirmed using Pearson's correlation coefficient analysis $(\mathrm{r}=-0.385$, $P=0.007$, Figure $1 C$ ) and Spearman's correlation coefficient analysis $(P=0.012)$.

\section{Expression of let-7a in NPC cell lines}

We first analyzed the expression level of let-7a in a panel of NPC cell lines with different degrees of differentiation and metastatic ability including CNE-1 (high differentiation), CNE-2(low differentiation), 5-8F (high metastatic ability), 6-10B (low metastatic ability), HONE-1(low differentiation). We observed that let-7a expression was relatively lower in CNE- 2 cells than in CNE-1 and HONE-1 cells, and also was lower in $5-8 \mathrm{~F}$ cells than in $6-10 \mathrm{~B}$ cells (Figure 2A), suggesting that let-7a expression may be associated with the degree of NPC cell differentiation and metastatic ability. Based on this expression pattern, we therefore chose CNE-2 and 5-8F cells for the following gain-of-function studies.

\section{Let-7a inhibited the protein expression of HMGA2 via binding to its 3 'UTR}

Based on the miRanda and TargetScan software, potential binding sites of let-7a in the 3'UTR of HMGA2 were predicted (Figure 2B). The successful overexpressions of let-7 $\mathrm{a}$ in the CNE-2 and 5-8F cells were confirmed by RT-qPCR (Figure 2C). We then performed a luciferase reporter assay to prove that let-7a directly targets HMGA2. We found that co-transfection of let-7a mimics and pLUCHMGA2-wt significantly decreased the luciferase activity in CNE-2 and 5-8F cells as compared with the control. Moreover, let-7a mimics had no effect on the luciferase activity when co-transfected with pLUC-HMGA2-mut (Figure 2D). These data showed that HMGA2 is one of direct targets of let-7a. We further analyzed the HMGA2 mRNA and protein expression by using RT-qPCR and western blotting respectively after transfecting CNE-2 and 5-8F cells with let-7a mimics. The increase let-7a levels significantly decreased HMGA2 protein expression at CNE-2 and 5-8F as determined by western blotting (Figure 2F, Additional 
A
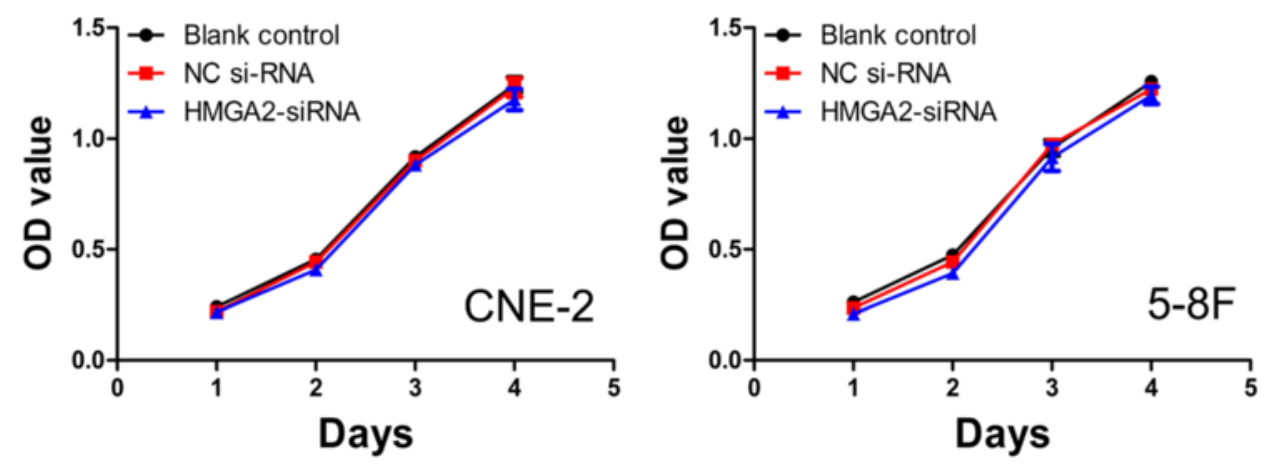

B

Blank control
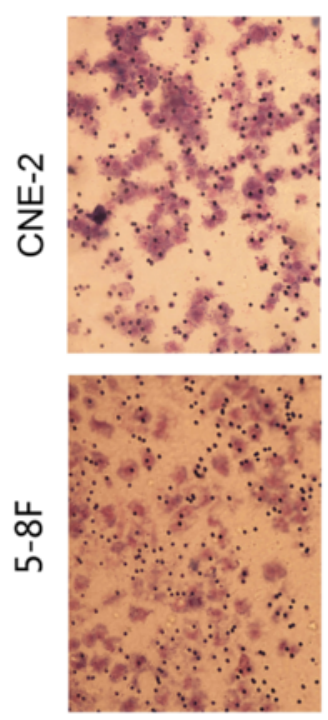

C
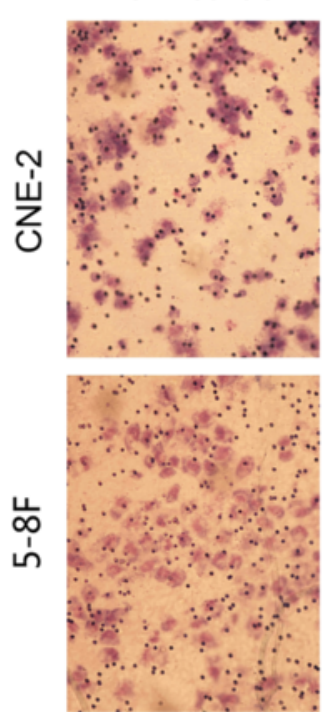

NC-siRNA
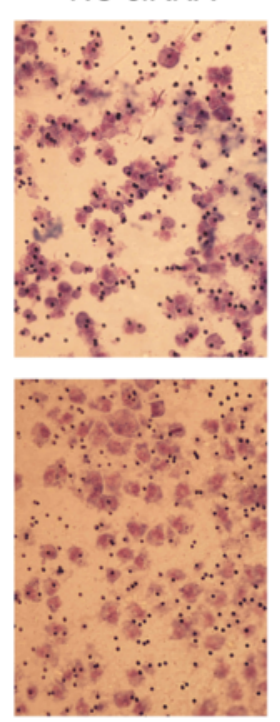

NC-siRNA
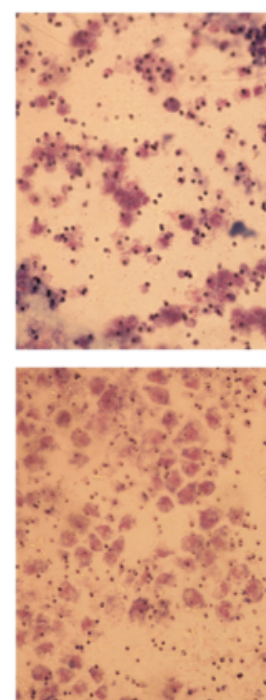

HMGA2-siRNA 2
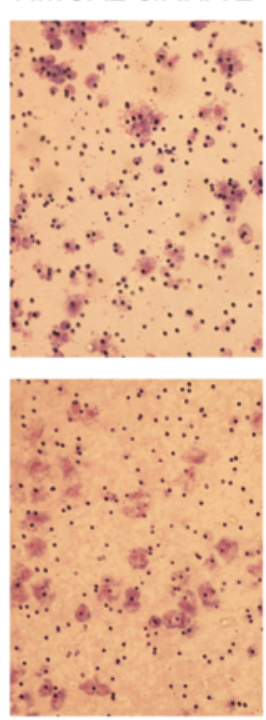

HMGA2-siRNA 2
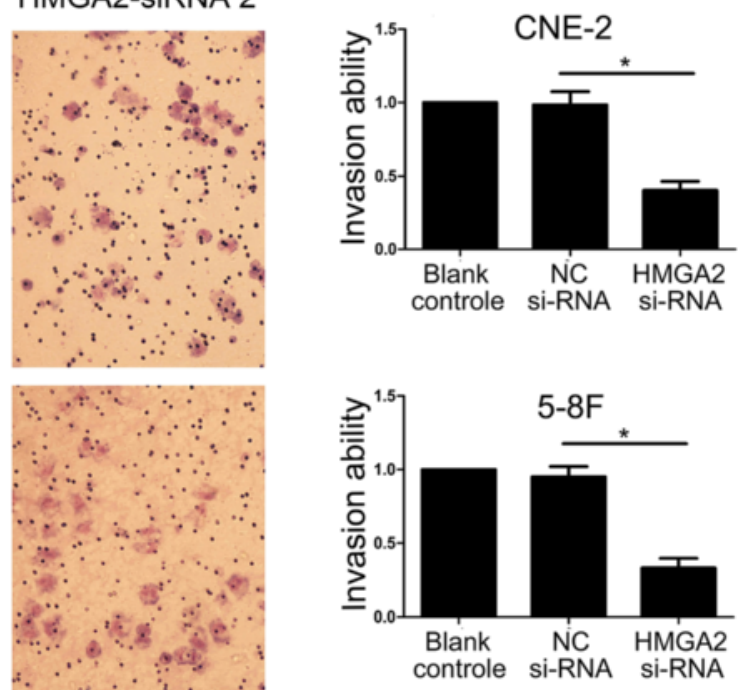

Figure 5 (See legend on next page.) 
(See figure on previous page.)

Figure $\mathbf{5}$ Knockdown of HMGA2 suppressed NPC cells migration and invasion. (A) The growth curves determined by MTT assay showed that knockdown of HMGA2 had no effect on NPC cells growth. (B) Down-regulated HMGA2 expression dramatically decreased the ability of CNE-2 and 5-8F cells migration in vitro. (C) Suppressed HMGA2 expression inhibited invasiveness of CNE-2 and 5-8F cells. The results were expressed as fold change relative to the corresponding blank control. Data is presented as the mean $\pm \mathrm{SD}$. ${ }^{*}, P<0.001$ compared with control.

file 2: Figure S1A), while mRNA remained unchanged (both $P>0.05$ ) (Figure 2E).

\section{HMGA2 is regulated uniaxially by let-7a, but not feedback regulatory loop}

The efficiency of HMGA2-siRNA was distinguished by RT-qPCR and confirmed by western blotting. As shown in Figure 3A-B, the relative expression of HMGA2 mRNA is the lower in the group of HMGA2-siRNA 2 after CNE-2 and 5-8F cells were transfected with si-HMGA2 for $48 \mathrm{~h}$, and HMGA2 protein expression of was significantly lower in CNE-2 and 5-8F cells transfected with the HMGA2siRNA 2 than with NC-siRNA or blank control. Interestingly, when we treated CNE-2 and 5-8F cells with a let-7a mimic, we found that the HMGA2 protein expression was significantly decreased, supposing whether HMGA2 was also able to regulate the expression of let-7a via a feedback loop in NPC cells. However, we found that knock-down HMGA2 had no effect on the let-7a expression at CNE-2 and $5-8$ F cells (Figure $3 \mathrm{C}$ ). Thus, there is no feedback regulatory loop between let-7a and HMGA2 in NPC cells.

\section{Let-7a suppressed NPC cells proliferation, migration and invasion}

We examined the effect of increased let-7a expression on NPC cell growth in vitro. The growth curves determined by MTT assay showed that let-7a inhibited NPC cell growth compared with negative control of let-7a (Figure 4A). To evaluate the impact of let-7a on cell migration and invasion, the cell migration and invasion assay were employed. In transwell migration assay, we found that overexpression of let-7a reduced $\mathrm{CNE}-2$ and $5-8 \mathrm{~F}$ cells migration (Figure $4 \mathrm{~B}, P<0.001$ ). Consistent with this finding, transwell invasion assay showed that let-7a mimics significantly inhibited invasion capacity of CNE-2 and 5-8F cells (Figure 4C, $P<0.001$ ).

\section{HMGA2 is involved in the regulation of cell migration and invasion by let-7a}

Because let-7a inhibits NPC cell proliferation, migration and invasion and suppresses HMGA2 protein expression, we are interested in exploring whether let-7a functions in cell proliferation, migration and invasion via targeting to HMGA2. To examine the role of HMGA2 in NPC cell migration and invasion, a siRNA against HMGA2 was introduced into CNE-2 and 5-8F cells to reduce HMGA2 expression (Figure 3B, Additional file 2: Figure S1B). HMGA2 silencing has no effect on
NPC cell growth (Figure 5A), but significantly decreased the migration and invasion of CNE-2 and 5-F8 cells (Figure 5B-C), which was similar to the phenotype of migration and invasion induced by let-7a (Figure 4B-C). To further verify whether let-7a regulated cell migration and invasion through HMGA2, we co-transfected let-7a mimics and HMGA2-siRNA into CNE-2 and 5-8F cells. We found that co-transfection of let-7a mimics and HMGA2-siRNA didn't profoundly reduced cell migration and invasion in NPC cells (Figure 6A-B). These findings suggested that HMGA2 is a functional mediator for let-7a in NPC cells.

\section{Let-7a and HMGA2 regulated epithelial-mesenchymal transition in NPC cells}

To further study the mechanism by which let-7a and HMGA2 regulate cell migration and invasion, we examined protein levels of EMT-associated genes in NPC CNE-2 and 5-8F cells with suppressed let-7a and HMGA2 expression respectively. We found that let-7a mimics decreased the expression of MMP2, MMP9 and EMTmarker genes including Snail, Slung, and Vimentin and increased E-cadherin and Zo-1 expression (Figure 6C, Additional file 2: Figure S1C). Similarly, knocking down endogenous HMGA2 expression suppressed the activation of MMP2, MMP9 and EMT-marker genes including Snail, Slug, and Vimentin and increased E-cadherin and Zo-1 expression, and elevated the expression of E-cadherin and Zo-1 (Figure 6C, Additional file 2: Figure S1C).

\section{Discussion}

Recognition of cancer-specific miRNAs and their targets is critical for understanding their roles in tumor development and progression, and may be significant for exploring novel therapeutic targets. Several reports indicated that miRNAs were abnormally expressed in NPC [28,29], and the dysregulated miRNAs could regulate NPC cell growth and metastasis [10-13]. Let-7a was reported to be frequently downregulated in several types of cancers, such as lung cancer [15], colon cancer [16], head and neck cancer [17], and pancreatic cancer [18]. Moreover, there were two studies shown the dysregulation of let-7a expression was involved in NPC cell proliferation and apoptosis [30,31], but little is known about the function and mechanism of let-7a involving in NPC metastasis.

Similar to a report from Cai et al. [31], we found that let-7a was downregulated in NPC clinical samples and further presented the evidence that let-7a expression 


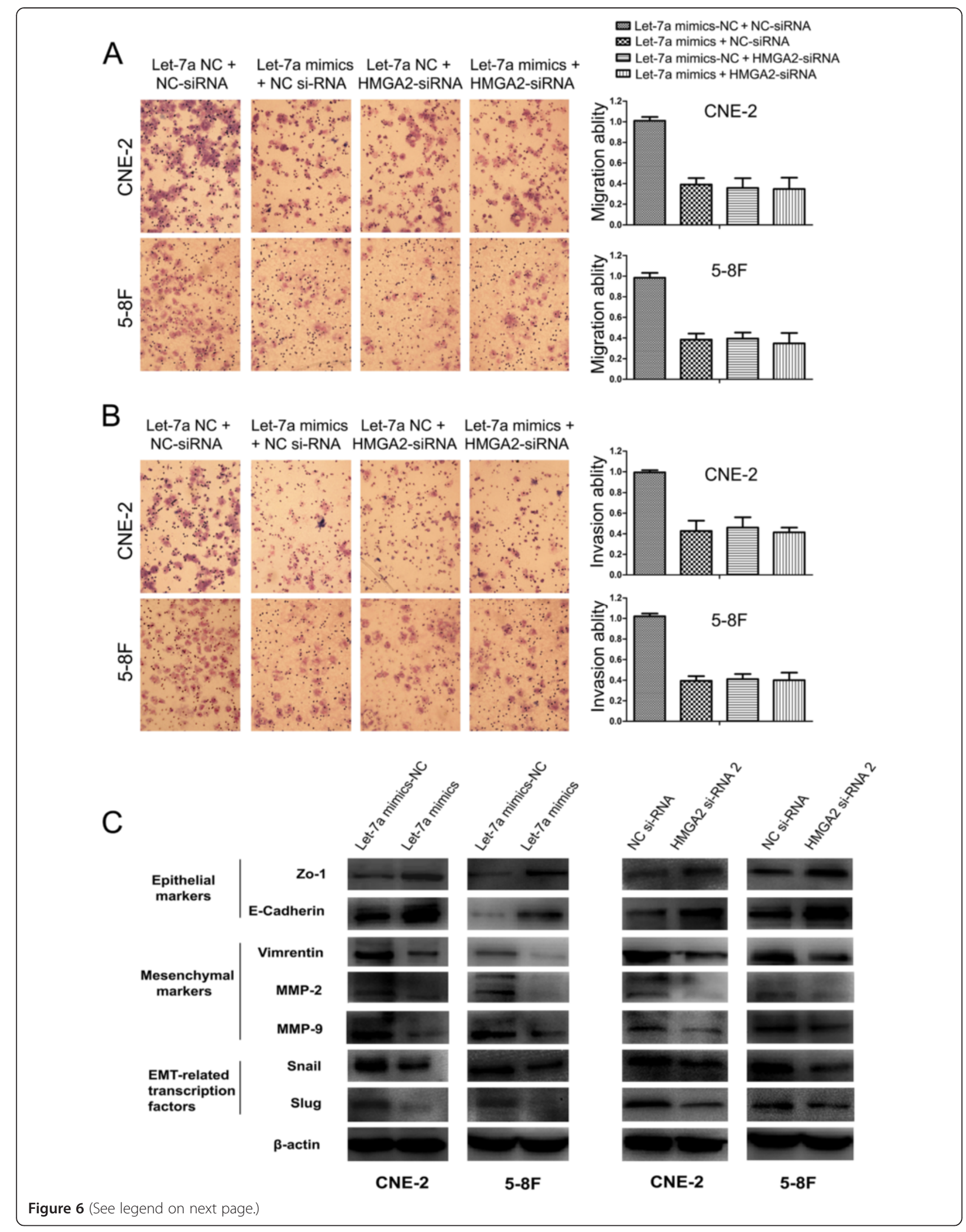


(See figure on previous page.)

Figure 6 HMGA2 is a functional target of let-7a to regulate NPC cells migration and invasion and expression of EMT-associated genes. (A) The migration assay suggested that co-transfection of let-7a mimics and HMGA2-siRNA didn't profoundly reduce NPC cells migration in comparison to HMGA2-siRNA+let-7a mimics-NC and NC-siRNA+let-7a mimics. (B) The invasion assay indicated that co-transfection of let-7a mimics and HMGA2-siRNA didn't further suppress thfce invasiveness of NPC cells compared with HMGA2-siRNA+let-7a mimics-NC and NC-siRNA+let-7a mimics. (C) Transfection of let-7a mimics reduced the expression of EMT-marker genes including Vimentin, MMP2, MMP9, Snail and Slug, and promoted E-cadherin and Zo-1 expression. Knocking down endogenous HMGA2 expression decreased the expression of EMT-marker genes including Vimentin, MMP2, MMP9, Snail and Slug, and increased E-cadherin and Zo-1 expression.

was positively correlated with the status of clinical stage, $\mathrm{T}$ classification, and $\mathrm{N}$ classification in NPC patients. Meanwhile, we firstly found HMGA2, which predicted as the target of let-7a, was overexpression in NPC tissues compared with normal nasopharyngeal epithelial tissues and correlated with the status of clinical stage and $\mathrm{N}$ classification, but no $\mathrm{T}$ classification. Moreover, there was an inverse correlation between let-7a expression and HMGA2 expression in NPC patient. Similarly, the significant inverse association was also detected between let7 and HMGA2 in esophageal cancer [21], retinoblastomas [32] and pituitary adenomas [33]. Therefore, we supposed that let-7a regulated NPC cell metastasis through targeting HMGA2.

In our study, synthetic let-7a mimics inhibited NPC cells migration and invasion and knockdown of HMGA2 was consistent with the effects of let-7a in NPC cells. Moreover, HMGA2 was identified as a direct and functional target of let-7a via binding to the 3 '-UTR of HMGA2. In lung cancer, Wang et al. reported that let-7a inhibited the proliferation and invasion of lung cancer cell line by inhibiting HMGA2 and K-RAS protein expression [34]. Recent studies have shown several members of let-7 family were diminished expression in breast cancer compared with normal breast tissues, and inhibited the breast cancer cell migration and invasive ability through regulating HMGA2, Lin28, GAB2, FN1, MAPK and MMPs [19,35,36]. Furthermore, we observed significantly diminished expression of let-7a only in protein level, but no mRNA level. Our results of the inconsistencies between the mRNA and protein levels of HMGA2 indicated that let-7a regulates HMGA2 expression at a posttranscriptional level, which is consistent with the mechanism of miRNAs [37]. Lin28 is also a target of let-7a and mainly plays an important role in cell stemness [38]. Interestingly, the double-negative feedback loop between let-7 and Lin28 was found in tumor cells [38]. Whether there is a double-negative feedback loop between let-7 and HMGA2 in NPC cells was an interesting problem. However, our results revealed knockdown of HMGA2 has no effect on the let-7a expression. Thus, there was just a unidirectional regulation between let-7a and HMGA2 in NPC cells.

Epithelial-mesenchymal transition (EMT) is a critical process by which epithelial cells lose their epithelial morphology and acquire a mesenchymal phenotype, characterized by the decrease of epithelial proteins such as E-cadherin and Zo-1, and the increase of mesenchymal proteins such as vimentin and fibronectin $[39,40]$. Snail and Slug are members of zinc finger family and play a central transcriptional role in the regulation of EMT by binding directly to specific E-boxes on the Ecadherin promoter $[41,42]$. It is widely accepted that EMT plays a significant role during tumor invasion and metastasis, and aggressive cancer cells often present with a loss of epithelial characteristics and acquire a mesenchymal phenotype [43]. Chang et al. reported that overexpression of let-7 effectively reversed the EMT phenotype, blocked migratory and invasive abilities in oral cancer cells [20]. Li et al. found that 3, 3'-diindolylmethane and isoflavone can cause up-regulation of let- 7 and miR-200 family members, leading to reversal of the EMT process in pancreatic cancer cells in vitro [44]. Similarly, our results indicated let-7a negatively modulates EMT process in NPC cells. HMGA2 is widely considered as a driver of tumor metastasis and a switching actor of EMT [45-47]. Consistent with the effect of let-7a mimics, knockdown of HMGA2 also suppressed EMT in NPC cells. Based on above result, we thought let-7a negatively modulates EMT process through targeting HMGA2 in NPC cells.

\section{Conclusions}

In summary, our study demonstrated that let-7a was downregulated and inversely associated with the clinical stage, $\mathrm{T}$ classification and $\mathrm{N}$ classification, and HMGA2 was upregulated and directly associated with the clinical stage and $\mathrm{N}$ classification in patients with NPC. Moreover, there was an inverse correlation between let-7a expression and HMGA2 expression in NPC patient. In addition, HMGA2 was negatively regulated at the posttranscriptional level by let-7a via a binding site of HMGA2-3'UTR. In addition, synthetic let-7a mimics suppressed NPC cells migration, invasion and EMT process and knockdown of HMGA2 was consistent with the effects of let-7a in NPC cells. This study suggests that let-7a/HMGA2 may play an important role in tumor metastasis and may be a novel diagnostic marker and potential therapeutic target in NPC. 


\section{Additional files}

Additional file 1: Figure S2. The luciferase reporter construct of pLUC. (hRluc: Rellina luciferase, hluc: Firefly luciferase, AMP: Ampicillin).

Additional file 2: Figure S1. Quantitative analysis of Western blot. (A) Let-7a mimics inhibited HMGA2 protein expression in NPC cells. (B) HMGA2 protein is reduced by small interfering RNA (HMGA2-siRNA 2) in NPC cells. (C) Let-7a and HMGA2 regulated the expression of MMPs and EMT-associated genes in NPC cells.

\section{Competing interests}

The authors declare that they have no competing interests.

\section{Authors' contributions}

ABW, KPW and ZXY designed the experiment, interpreted the data and prepared the manuscript. KPW, ABW, JML, YLM, YML, XS, YZW, SJL and LLX conducted the experiment, collected the data and helped to prepare the manuscript. All authors read and approved the final manuscript.

\section{Acknowledgements}

This work was supported by the funding from National Natural Science Foundation of China (81201672), the Doctoral Program of Affiliated Hospital of Guangdong Medical College (No. BK201208) and the Science and Technology Planning Project of Guangdong Province (No. 2013B021800078).

Received: 7 January 2015 Accepted: 12 March 2015

Published online: 31 March 2015

\section{References}

1. Jemal A, Bray F, Center MM, Ferlay J, Ward E, Forman D. Global cancer statistics. Cancer J Clinicians. 2011:61(2):69-90.

2. Wei WI, Sham JS. Nasopharyngeal carcinoma. Lancet. 2005;365(9476):2041-54

3. Bartel DP. MicroRNAs: genomics, biogenesis, mechanism, and function. Cell. 2004;116(2):281-97.

4. He L, Hannon GJ. MicroRNAs: small RNAs with a big role in gene regulation. Nat Rev Genet. 2004;5(7):522-31.

5. Zamore PD, Haley B. Ribo-gnome: the big world of small RNAs. Science (New York, NY). 2005;309(5740):1519-24

6. Ambros V. MicroRNA pathways in flies and worms: growth, death, fat, stress, and timing. Cell. 2003;113(6):673-6.

7. Ambros V. The functions of animal microRNAs. Nature. 2004;431(7006):350-5.

8. Esquela-Kerscher A, Slack FJ. Oncomirs - microRNAs with a role in cancer. Nat Rev Cancer. 2006;6(4):259-69.

9. Zhang B, Pan X, Cobb GP, Anderson TA. microRNAs as oncogenes and tumor suppressors. Dev Biol. 2007;302(1):1-12.

10. Liu Z, Yang H, Luo W, Jiang Q, Mai C, Chen Y, et al. Loss of cytoplasmic KLF4 expression is correlated with the progression and poor prognosis of nasopharyngeal carcinoma. Histopathology. 2013;63(3):362-70.

11. Yi C, Wang Q, Wang L, Huang Y, Li L, Liu L, et al. MiR-663, a microRNA targeting p21(WAF1/CIP1), promotes the proliferation and tumorigenesis of nasopharyngeal carcinoma. Oncogene. 2012:31(41):4421-33.

12. Lu J, He ML, Wang L, Chen Y, Liu X, Dong Q, et al. MiR-26a inhibits cell growth and tumorigenesis of nasopharyngeal carcinoma through repression of EZH2. Cancer Res. 2011;71(1):225-33.

13. Liu Z, Long X, Chao C, Yan C, Wu Q, Hua S, et al. Knocking down CDK4 mediates the elevation of let-7c suppressing cell growth in nasopharyngeal carcinoma. BMC Cancer. 2014;14(1):274.

14. Bussing I, Slack FJ, Grosshans H. let-7 microRNAs in development, stem cells and cancer. Trends Mol Med. 2008;14(9):400-9.

15. Pan L, Gong Z, Zhong Z, Dong Z, Liu Q, Le Y, et al. Lin-28 reactivation is required for let-7 repression and proliferation in human small cell lung cancer cells. Mol Cell Biochem. 2011:355(1-2):257-63.

16. King CE, Wang L, Winograd R, Madison BB, Mongroo PS, Johnstone CN, et al. LIN28B fosters colon cancer migration, invasion and transformation through let-7-dependent and -independent mechanisms. Oncogene. 2011:30(40):4185-93.

17. Tu HF, Lin SC, Chang KW. MicroRNA aberrances in head and neck cancer: pathogenetic and clinical significance. Curr Opin Otolaryngol Head Neck Surg. 2013;21(2):104-11.
18. Ali S, Almhanna K, Chen W, Philip PA, Sarkar FH. Differentially expressed miRNAs in the plasma may provide a molecular signature for aggressive pancreatic cancer. Am J Transl Res. 2010;3(1):28-47.

19. Guo L, Chen C, Shi M, Wang F, Chen X, Diao D, et al. Stat3-coordinated Lin-28-let7-HMGA2 and miR-200-ZEB1 circuits initiate and maintain oncostatin M-driven epithelial-mesenchymal transition. Oncogene. 2013;32(45):5272-82.

20. Chang CJ, Hsu CC, Chang CH, Tsai LL, Chang YC, Lu SW, et al. Let-7d functions as novel regulator of epithelial-mesenchymal transition and chemoresistant property in oral cancer. Oncol Rep. 2011;26(4):1003-10.

21. Liu Q, Liu T, Zheng S, Gao X, Lu M, Sheyhidin I, et al. HMGA2 is down-regulated by microRNA let-7 and associated with epithelial-mesenchymal transition in oesophageal squamous cell carcinomas of Kazakhs. Histopathology. 2014:65(3):408-17.

22. Hock R, Furusawa T, Ueda T, Bustin M. HMG chromosomal proteins in development and disease. Trends Cell Biol. 2007;17(2):72-9.

23. Sgarra R, Rustighi A, Tessari MA, Di Bernardo J, Altamura S, Fusco A, et al. Nuclear phosphoproteins HMGA and their relationship with chromatin structure and cancer. FEBS Lett. 2004;574(1-3):1-8.

24. Fusco A, Fedele M. Roles of HMGA proteins in cancer. Nat Rev Cancer. 2007;7(12):899-910.

25. Lee YS, Dutta A. The tumor suppressor microRNA let-7 represses the HMGA2 oncogene. Genes Dev. 2007;21(9):1025-30.

26. Nishida N, Mimori K, Fabbri M, Yokobori T, Sudo T, Tanaka F, et al. MicroRNA-125a-5p is an independent prognostic factor in gastric cancer and inhibits the proliferation of human gastric cancer cells in combination with Trastuzumab. Clin Cancer Res. 2011;17(9):2725-33.

27. linuma $\mathrm{H}$. Clinicopathological and prognostic significance of microRNA-107 and its relationship to DICER1 mRNA expression in gastric cancer. Oncol Rep. 2012;27(6):1759-64.

28. Chen HC, Chen GH, Chen YH, Liao WL, Liu CY, Chang KP, et al. MicroRNA deregulation and pathway alterations in nasopharyngeal carcinoma. $\mathrm{Br} \mathrm{J}$ Cancer. 2009;100(6):1002-11.

29. Liu N, Chen NY, Cui RX, Li WF, Li Y, Wei RR, et al. Prognostic value of a microRNA signature in nasopharyngeal carcinoma: a microRNA expression analysis. Lancet Oncol. 2012;13(6):633-41.

30. Wong TS, Man OY, Tsang CM, Tsao SW, Tsang RK, Chan JY, et al. MicroRNA let-7 suppresses nasopharyngeal carcinoma cells proliferation through downregulating c-Myc expression. J Cancer Res Clin Oncol. 2011;137(3):415-22.

31. Cai K, Wan Y, Sun G, Shi L, Bao X, Wang Z. Let-7a inhibits proliferation and induces apoptosis by targeting EZH2 in nasopharyngeal carcinoma cells. Oncol Rep. 2012;28(6):2101-6.

32. Mu G, Liu H, Zhou F, Xu X, Jiang H, Wang Y, et al. Correlation of overexpression of HMGA1 and HMGA2 with poor tumor differentiation, invasion, and proliferation associated with let-7 down-regulation in retinoblastomas. Hum Pathol. 2010:41(4):493-502.

33. Qian ZR, Asa SL, Siomi H, Siomi MC, Yoshimoto K, Yamada S, et al. Overexpression of HMGA2 relates to reduction of the let-7 and its relationship to clinicopathological features in pituitary adenomas. Modern Pathol. 2009:22(3):431-41.

34. Wang $Y Y$, Ren T, Cai YY, He XY. MicroRNA let-7a inhibits the proliferation and invasion of nonsmall cell lung cancer cell line 95D by regulating K-Ras and HMGA2 gene expression. Cancer Biother Radiopharm. 2013;28(2):131-7.

35. Dangi-Garimella S, Yun J, Eves EM, Newman M, Erkeland SJ, Hammond SM, et al. Raf kinase inhibitory protein suppresses a metastasis signalling cascade involving LIN28 and let-7. EMBO J. 2009:28(4):347-58.

36. Qian P, Zuo Z, Wu Z, Meng X, Li G, Wu Z, et al. Pivotal role of reduced let-7 g expression in breast cancer invasion and metastasis. Cancer Res. 2011;71(20):6463-74.

37. Mayr C, Hemann MT, Bartel DP. Disrupting the pairing between let-7 and Hmga2 enhances oncogenic transformation. Science (New York, NY) 2007;315(5818):1576-9.

38. Yang X, Lin X, Zhong X, Kaur S, Li N, Liang S, et al. Double-negative feedback loop between reprogramming factor LIN28 and microRNA let-7 regulates aldehyde dehydrogenase 1-positive cancer stem cells. Cancer Res. 2010;70(22):9463-72.

39. Kalluri $R$, Weinberg RA. The basics of epithelial-mesenchymal transition. J Clin Invest. 2009;119(6):1420-8.

40. Kalluri R, Neilson EG. Epithelial-mesenchymal transition and its implications for fibrosis. J Clin Invest. 2003:112(12):1776-84

41. Moreno-Bueno G, Cubillo E, Sarrio D, Peinado H, Rodriguez-Pinilla SM, Villa $\mathrm{S}$, et al. Genetic profiling of epithelial cells expressing E-cadherin repressors 
reveals a distinct role for Snail, Slug, and E47 factors in epithelial-mesenchymal transition. Cancer Res. 2006;66(19):9543-56.

42. Castro Alves C, Rosivatz E, Schott C, Hollweck R, Becker I, Sarbia M, et al. Slug is overexpressed in gastric carcinomas and may act synergistically with SIP1 and Snail in the down-regulation of E-cadherin. J Pathol. 2007;211(5):507-15.

43. Scheel C, Weinberg RA. Phenotypic plasticity and epithelial-mesenchymal transitions in cancer and normal stem cells? Int J Cancer J Int du Cancer. 2011:129(10):2310-4.

44. Li Y, VandenBoom 2nd TG, Kong D, Wang Z, Ali S, Philip PA, et al. Up-regulation of miR-200 and let-7 by natural agents leads to the reversal of epithelial-tomesenchymal transition in gemcitabine-resistant pancreatic cancer cells. Cancer Res. 2009;69(16):6704-12.

45. Thuault S, Tan EJ, Peinado H, Cano A, Heldin CH, Moustakas A. HMGA2 and Smads Co-regulate SNAIL1 expression during induction of epithelialto-mesenchymal transition. J Biol Chemistry. 2008:283(48):33437-46.

46. Thuault S, Valcourt U, Petersen M, Manfioletti G, Heldin CH, Moustakas A. Transforming growth factor-beta employs HMGA2 to elicit epithelialmesenchymal transition. J Cell Biol. 2006;174(2):175-83.

47. Morishita A, Zaidi MR, Mitoro A, Sankarasharma D, Szabolcs M, Okada Y, et al. HMGA2 is a driver of tumor metastasis. Cancer Res. 2013;73(14):4289-99.

\section{Submit your next manuscript to BioMed Central and take full advantage of:}

- Convenient online submission

- Thorough peer review

- No space constraints or color figure charges

- Immediate publication on acceptance

- Inclusion in PubMed, CAS, Scopus and Google Scholar

- Research which is freely available for redistribution 